\title{
Pie diabético en México: factores de riesgo para mortalidad posterior a una amputación mayor, a 5 años, en un hospital de salud pública de segundo nivel
}

\author{
Diabetic foot in Mexico: risk factors for mortality after a major amputation, at 5 years, \\ in a second-level public health hospital
}

José E. Martínez-Escalante ${ }^{1 *}$, M. Elena Romero-lbargüengoitia1, Heliodoro Plata-Álvarez², Gilberto López-Betancourt ${ }^{1}$, Rodrigo Otero-Rodríguez ${ }^{1}$, Alan A. Garza-Cantús y Mónica E. Corral-Guerrero ${ }^{4}$

${ }^{1}$ Hospital Alta Especialidad, Christus Muguerza, Monterrey, Nuevo León, México; ${ }^{2}$ Hospital General No. 6, Monterrey, Nuevo León, México; ${ }^{3}$ Servicio de Coloproctología, Hospital General de México, Ciudad de México, México; ${ }^{4}$ Laredo Medical Center, Laredo, Texas, Estados Unidos de América

\section{Resumen}

Objetivo: Investigar de manera retrospectiva a 5 años el porcentaje de mortalidad en los pacientes operados de amputación mayor por pie diabético e identificar los factores de riesgo asociados que aumentan la mortalidad en la población mexicana. Método: Estudio retrospectivo de pacientes sometidos a amputación mayor por pie diabético del 1 de enero al 31 de diciembre de 2009 en un hospital de segundo nivel. Resultados: Se incluyeron en el protocolo de estudio 37 pacientes que cumplían los criterios de inclusión, y se encontró que 10 (27.03\%) continuaban con vida y 27 (72.97\%) habían fallecido. Los pacientes a quienes se realizó una amputación y tenían tres o más enfermedades concomitantes mostraron un riesgo 1.6 veces más alto de morir $(p=0.018)$. Cuanto mayor era la glucemia previa al momento de la amputación, mayor fue la probabilidad de muerte a 5 años $(p=0.015)$. Conclusiones: En México hacen falta estudios con seguimiento prospectivo, de carácter multicéntrico, con una muestra heterogénea, que permitan tener un panorama nacional.

Palabras clave: Amputación infracondílea. Amputación supracondílea. Mortalidad en amputación mayor. Pie diabético.

\begin{abstract}
Objective: To investigate retrospectively at 5 years the mortality rate in postoperative patients of major amputation secondary to diabetic foot and to identify the associated risk factors that increase mortality in the Mexican population. Method: Retrospective study that included patients who underwent major amputation secondary to diabetic foot from January 1 to December 31, 2009 in a second-level hospital. Results: 37 patients who met the inclusion criteria were included in the study protocol, finding that 10 patients (27.03\%) were still alive and 27 patients (72.97\%) had died. Observing In patients who undergo an amputation and have three or more comorbidities, they have a 1.6 times higher risk of dying $(p=0.018)$ and that the higher the glycemia prior to the amputation, the greater the probability of dying at 5 years $(p=0.015)$. Conclusions:
\end{abstract}

\section{Correspondencia:}

*José E. Martínez-Escalante

Sacramento, 205a

Col. Mitras Centro

Fecha de recepción: 21-03-2020

C.P. 64460 , Monterrey, N.L., México

E-mail: emmanuelmtz @ hotmail.es

0009-7411/@ 2020 Academia Mexicana de Cirugía. Publicado por Permanyer. Este es un artículo open access bajo la licencia CC BY-NC-ND (http://creativecommons.org/licenses/by-nc-nd/4.0/). 
Studies are needed in Mexico with prospective follow-up, multicenter in nature, with a heterogeneous sample, which allows us to have a National panorama.

Key words: Infracondylar amputation. Supracondylar amputation. Mortality in major amputation. Diabetic foot.

\section{Introducción}

El pie diabético es una complicación de la diabetes asociada con una gran morbilidad, una elevada mortalidad, granes costos y una calidad de vida reducida. Generalmente se presenta como úlceras de pie diabético con o sin un proceso infeccioso subyacente y pie de Charcot en presencia de neuropatía periférica o enfermedad arterial periférica en personas con diabetes ${ }^{1}$, y es el precursor más importante para las amputaciones de las extremidades inferiores $^{1,2}$. La prevalencia global de la enfermedad del pie diabético se estima alrededor del $6 \% \%^{1,3}$. Un estudio de cohorte basado en la población en el Reino Unido demostró que el desarrollo de una úlcera de pie diabético se asocia con una mortalidad del $5 \%$ en los primeros 12 meses y del $42 \%$ en 5 años; además, estos pacientes tienen un riesgo de muerte 2.5 veces mayor que los pacientes diabéticos sin heridas en los pies. Como factores de riesgo se han descrito la edad avanzada, el sexo masculino y el tabaquis$\mathrm{mo}^{4-6}$. Un paciente diabético tiene un riesgo de amputación de 15 a 40 veces mayor que una persona que no es diabética?

Las tasas de amputaciones de la extremidad inferior secundarias a pie diabético fueron de $8.3,9.5 \mathrm{y}$ 9.2 por 100,000 en la población general en México en los años 2009, 2010 y 2011, respectivamente ${ }^{8}$.

En muchos casos es difícil la elección del nivel de amputación, el cual es importante para la recuperación de la extremidad amputada, ya que en general son pacientes difíciles de rehabilitar y que además sufren una mortalidad significativa por problemas médicos que con frecuencia preceden a la curación definitiva de la herida. Se ha observado que, cuando se realiza una amputación infracondílea, hasta el $75 \%$ los pacientes mayores nunca pueden volver a deambular y tienen más problemas de curación que requieren conversión a amputación supracondílea en un $20 \%$ adicional de pacientes ${ }^{9}$.

La mortalidad a 30 días después de la amputación en los pacientes con pie diabético y diabetes mellitus tipo 2 es del $8.6 \%$, entre el $13 \%$ y el $40 \%$ en 1 año, del $35 \%$ al $65 \%$ en 3 años y del $39 \%$ al $80 \%$ en 5 años, siendo peor que para la mayoría de los tumores malignos ${ }^{7,10,11}$. Estos porcentajes pueden aumentar en los países en desarrollo, ya que los pacientes tienden a buscar atención médica cuando la infección de la úlcera está avanzada, lo que incrementa el riesgo de amputación y muerte ${ }^{10}$.

Además de la edad (hazard ratio [HR] por año: 1.08), el sexo masculino (HR: 1.65; intervalo de confianza del 95\% [IC 95\%]: 1.18-2.32; $p=0.004)$, la enfermedad renal crónica (HR: 1.83), la diálisis (HR: 6.43) y la enfermedad arterial periférica (HR: 1.44) son variables predictivas independientes de muerte ${ }^{6,12}$.

Los pacientes con enfermedad renal en etapa terminal que reciben diálisis tienen un riesgo especialmente alto de amputación, encontrando en un estudio que en el año 2014 se amputaron el 2.66\% [IC 95\%: 2.59-2.72] de estos pacientes ${ }^{13}$. Las tasas ajustadas de mortalidad a 1 año de la amputación de la extremidad inferior para los pacientes con enfermedad renal terminal que reciben diálisis disminuyeron del $52.2 \%$ (IC 95\%: 50.953.4) en 2000 al 43.6\% (IC 95\%: 42.5-44.8) en $2013^{13}$. En otro estudio se halló que la mortalidad hospitalaria en el posquirúrgico temprano en los pacientes operados de amputación que se encontraban en diálisis fue mayor $(7.7 \%$ vs. $2.9 \%$; $p<0.001)$, y los eventos de infarto de miocardio fueron marginalmente más altos ( $2.4 \%$ vs. $1.6 \% ; p=0.053$ ) que en los pacientes $\sin$ diálisis ${ }^{14}$.

En otro estudio, el $39.7 \%$ de los pacientes tenían una amputación supracondílea y el $61.1 \%$ de ellos fallecieron. Estos pacientes mostraron un riesgo de muerte 4.5 veces mayor que el de los pacientes que tuvieron amputaciones a otros niveles?

Más del $50 \%$ de los pacientes sometidos a una primera amputación mayor tienen evidencia de enfermedad arterial periférica ${ }^{6}$.

Faglia, et al. ${ }^{5}$ encontraron que el sexo femenino se asociaba independientemente con la muerte (HR: 1.96; IC 95\%: 1.08-3.56; $p=0.027$ ). En los países desarrollados, más de la mitad de las muertes posteriores a la amputación son causadas por eventos cardiovasculares y cerebrovasculares. En contraste, los estudios sobre la mortalidad de pacientes amputados con pie diabético en países en desarrollo y recientemente desarrollados informan una proporción mucho mayor de muertes por afecciones sépticas ${ }^{6}$. 
En un metaanálisis realizado en 2017 se encontró que los factores de riesgo clínicos asociados con una mayor mortalidad incluían la diabetes mellitus tipo 2 (odds ratio [OR]: 2.02-2.20), la enfermedad arterial periférica (OR: 2.34), la enfermedad de la arteria coronaria (OR: 2.01-3.33), la enfermedad cerebrovascular (OR: 2.50-2.55), la enfermedad renal terminal y la dependencia de diálisis (OR: 2.12-5.35), la clase $\geq 4$ de la American Society of Anesthesiologists (ASA) (OR: 2.04), la demencia (OR: 2.02-2.77) y el estado no ambulatorio (OR: 2.28). Los factores quirúrgicos, incluidos un nivel de amputación más alto (OR: 2.43.2) y la necesidad de cirugía por etapas con amputación mayor inicial (OR: 2.49), también se correlacionaron con una mayor mortalidad ${ }^{15}$. Los factores independientemente asociados con la muerte fueron la edad avanzada (OR: 1.29), los antecedentes de insuficiencia cardiaca (OR: 1.71), la enfermedad renal (OR: 1.84) y la enfermedad pulmonar obstructiva crónica (EPOC) (OR: 1.3)

En otro estudio, la falta de creación de prótesis fue un factor independiente de mortalidad (OR: 2.60; IC 95\%: $1.16-6.25 ; p=0.028)^{5}$.

Son escasos los datos a largo plazo con respecto a la mortalidad de los pacientes operados de amputación mayor secundaria a pie diabético en México8. Se necesitan con urgencia estudios que busquen información más detallada sobre afecciones clínicas y quirúrgicas, causas específicas de mortalidad y costos indirectos, para mejorar el conocimiento de la mortalidad y de la carga económica de esta complicación de la diabetes en el país ${ }^{8}$.

El propósito principal de nuestro estudio fue investigar de manera retrospectiva a 5 años el porcentaje de mortalidad en los pacientes con diabetes mellitus tipo 2 sometidos a amputación mayor secundaria a pie diabético, y el objetivo secundario fue identificar los factores de riesgo asociados que aumentan la mortalidad en la población mexicana.

\section{Método}

La cohorte retrospectiva consistió en todos los sujetos que se sometieron a una amputación mayor secundaria a pie diabético desde el 1 de enero de 2009 hasta el 31 de diciembre de 2009 en el Hospital General de Zona No. 6 de Monterrey, Nuevo León, México. Los pacientes fueron identificados en el registro de amputaciones mayores del programa de heridas crónicas del hospital, y se incluyeron en el estudio todos aquellos que tenían expediente clínico completo, presentaban diabetes mellitus tipo 2 según la historia clínica y, en caso de no tener acta de defunción en el expediente de la clínica a la que correspondían o en el propio hospital, se les pudiera localizar en su vivienda o a través de algún familiar que diera fe de que continuaba con vida. El estudio fue revisado y aprobado por el Comité Ético del Hospital General de Zona No. 6 y se recabó el consentimiento informado de todos los familiares de pacientes en el momento de la encuesta, acudiendo al total de las viviendas de los pacientes y descartando por conveniencia a los que no fueron localizables. Se recopilaron los datos de si continuaba o no con vida, la edad en el momento de la amputación, el sexo, la clínica a la cual pertenecía, los meses de supervivencia tras la amputación, la etiología de la muerte tomada del acta de defunción y de la amputación tomada del expediente clínico, el tipo de amputación, si pertenecía al programa ATHODO IMSS (el cual brinda atención médica a domicilio), el tiempo desde el diagnóstico de la enfermedad hasta el momento de la amputación, el último reporte de glucosa capilar registrado en el expediente previo a la amputación, la comorbilidad tomada del expediente clínico, si tenía o no registro de curaciones, si llevaba o no dieta para diabético, y si contaba con amputación mayor previa del miembro contralateral. Posterior a la recolección de la información, la base de datos fue anonimizada y exportada a un software estadístico.

Se utilizaron la mediana y el rango intercuartílico para las variables continuas no distribuidas normalmente, la media y la desviación estándar para las variables continuas distribuidas normalmente, y el porcentaje para las variables discretas. Se analizaron variables discretas con la prueba de ji al cuadrado y, cuando fue apropiado, la prueba exacta de Fisher, y variables cuantitativas no distribuidas normalmente usando la prueba U no paramétrica de Mann-Whitney. Se realizó un análisis multivariable para identificar los predictores independientes de mortalidad perioperatoria con análisis de regresión logística. Se estimaron las OR y los IC 95\%, y se estableció un valor de $p<0.05$ como umbral para la significación estadística. El análisis estadístico se llevó a cabo con el programa SPSS versión 25 para Windows (SPSS Inc., Chicago, IL, USA).

\section{Resultados}

De los 66 pacientes que pertenecían al programa de amputaciones referente al año 2009 se incluyeron 
Tabla 1. Características de la población

\begin{tabular}{|c|c|c|c|c|c|c|}
\hline Variable & & Vivos & Muertos & OR vivo & OR muerto & $p$ - valor \\
\hline \multirow[t]{3}{*}{ Genero } & & & & & & 1 \\
\hline & Masculino & $7(28.0 \%)$ & $18(72.0 \%)$ & & 1.16 & \\
\hline & Femenino & $3(25 \%)$ & $9(75 \%)$ & & 1.05 & \\
\hline \multicolumn{7}{|l|}{ Etiología } \\
\hline & Infecciosa & $4(16.7 \%)$ & $20(83.3 \%)$ & 0.54 & 2.3 & 0.118 \\
\hline & Vascular & $10(32.3 \%)$ & $21(67.7 \%)$ & & 1.2867 & 0.162 \\
\hline & Neuropática & $5(38.5 \%)$ & $8(61.5 \%)$ & 1.68 & 0.711 & 0.275 \\
\hline \multirow[t]{3}{*}{ Tipo de amputación } & & & & & & 0.275 \\
\hline & Supracondílea & $9(26.5 \%)$ & $25(73.5 \%)$ & & 1.35 & \\
\hline & Infracondílea & $1(33.3 \%)$ & $2(66.7 \%)$ & & 0.72 & \\
\hline \multicolumn{7}{|l|}{ Comorbilidades } \\
\hline \multirow[t]{2}{*}{ HAS } & Con HAS & $8(29.6 \%)$ & $19(70.4 \%)$ & 1.13 & 0.675 & 0.694 \\
\hline & Sin HAS & $2(20 \%)$ & $8(80 \%)$ & & & \\
\hline \multirow[t]{2}{*}{ ERC } & Con ERC & $4(20 \%)$ & $16(80 \%)$ & 0.675 & 0.297 & 0.46 \\
\hline & Sin ERC & $6(35.3)$ & $11(64.7)$ & & & \\
\hline \multirow[t]{2}{*}{ FA } & Con FA & $0(0 \%)$ & $1(100 \%)$ & & 1.038 & 1 \\
\hline & Sin FA & $10(27.2 \%)$ & $26(72.2 \%)$ & & & \\
\hline \multirow[t]{2}{*}{ ICC } & Con ICC & $0(0 \%)$ & $8(100 \%)$ & & 1.421 & 0.79 \\
\hline & Sin ICC & $10(34.5 \%)$ & $19(65.5 \%)$ & & & \\
\hline \multirow[t]{2}{*}{ EVC } & Con EVC & $0(0 \%)$ & $2(100 \%)$ & & 1.08 & 1 \\
\hline & Sin EVC & $10(28.6 \%)$ & $25(71.4 \%)$ & & & \\
\hline \multirow[t]{2}{*}{ EPOC } & Con EPOC & $0(0 \%)$ & $3(100 \%)$ & & 1.125 & 0.54 \\
\hline & Sin EPOC & $10(29.48 \%)$ & $24(70.6 \%)$ & & & \\
\hline \multirow[t]{2}{*}{ IAM } & Con IAM & $1(11.1 \%)$ & 8 (88.9\%) & 0.338 & 1.279 & 0.393 \\
\hline & Sin IAM & $9(32.1 \%)$ & $19(67.9 \%)$ & & & \\
\hline \multirow[t]{2}{*}{3 o más comorbilidades } & Más de 3 & $0(0 \%)$ & $11(100 \%)$ & & 1.688 & 0.018 \\
\hline & Menos de 3 & $10(38.5 \%)$ & $16(61.5 \%)$ & & & \\
\hline \multirow[t]{2}{*}{ AMP PREV } & Sí & $0(0 \%)$ & $7(100 \%)$ & & 1.35 & 0.155 \\
\hline & No & $10(33.3 \%)$ & $20(66.7 \%)$ & & & \\
\hline
\end{tabular}

Las variables nominales se expresan como frecuencia total (\%) y las variables cuantitativas de distribución normal como media \pm desviación estándar.

AMP PREV: Amputación mayor previa de la pierna contralateral; EPOC: enfermedad pulmonar obstructiva crónica; ERC: enfermedad renal crónica; EVC: evento vascular cerebral; FA: fibrilación auricular; HAS: hipertensión arterial sistémica; IAM: infarto agudo al miocardio; ICC: insuficiencia cardiaca congestiva; OR: odds ratio.

en el protocolo de estudio 37 que cumplían con los criterios de inclusión. De estos 37 pacientes, se encontró que 10 (27.03\%) continuaban con vida y 27 (72.97\%) habían fallecido. De la totalidad de pacientes, 25 eran hombres, de los cuales 18 (72\%) habían fallecido (OR: 1.16), y 12 eran mujeres, de las cuales $9(75 \%)$ habían fallecido (OR: 1.05) (Tabla 1).

La mortalidad por años fue del $8.1 \%$ antes del primer año, el $16.2 \%$ antes del segundo, el $18.9 \%$ antes del tercero, el $18.9 \%$ antes del cuarto y el $10.8 \%$ antes 
del quinto; y sumada es del $8.1 \%$ antes del primer año, del $43 \%$ a los 3 años y del $72.97 \%$ a los 5 años.

En los 27 pacientes que fallecieron, las causas de la muerte en orden de frecuencia fueron choque séptico en 12, cardiopatía isquémica en 3 , descompensación de la diabetes en 8 , enfermedad renal crónica en 3 y enfermedad cerebrovascular en 1 paciente.

Se encontró que, en cuanto al motivo de la amputación, habían fallecido 20 pacientes a quienes se les amputó por etiología infecciosa (83.3\%; OR: 2.3; $p=$ 0.118), 21 pacientes de etiología vascular (67.7\%; OR: 1.28; $p$ 0.162) y 8 pacientes de etiología neuropática (61.5\%; OR: 0.711; $p=0.275$ ).

Al buscar la relación entre el tipo de amputación y la muerte de los pacientes se observó que, de aqueIlos con una amputación supracondílea, 25 (73.5\%; OR: 0.72) habían muerto, y con amputación infracondílea habían muerto 2 (66.7\%; $p=0.275)$.

Al observar la comorbilidad se encontró que murieron 19 pacientes (70.4\%; OR: 0.675; $p=0.694$ ) con hipertensión arterial sistémica, 16 pacientes $(80 \%$; OR: 1.47) con enfermedad renal crónica, un paciente con fibrilación auricular (OR: 1.038; $p$ 1.038), 8 pacientes con insuficiencia cardiaca (OR: $1.421 ; p=0.79$ ), 2 pacientes con enfermedad cerebrovascular (OR: 1.08; $p=1.0$ ), 3 pacientes con EPOC (OR: 1.125; $p=0.54)$ y 8 pacientes (88.9\%; OR: $1.279 ; p=0.39$ ) con infarto agudo al miocardio.

Al buscar de manera intencionada los pacientes con tres o más enfermedades concomitantes y relacionarlos con los pacientes vivos y muertos se encontró que ningún paciente $(0 \%)$ con más de tres enfermedades concomitantes continuaba con vida y que $11(100 \%)$ habían fallecido, y con menos de tres enfermedades concomitantes se halló que 10 pacientes $(38.5 \%)$ continuaban con vida y $16(61.5 \%)$ estaban muertos. Se realizó la prueba de Fisher, que demostró, para los pacientes finados, significancia estadística con $p=0.018$ y OR de 1.688 .

Ningún paciente de los siete con amputación mayor bilateral continuaba con vida (OR: 1.35; $p=0.15$ ).

Se analizó la última glucemia previa a la amputación en los pacientes vivos y muertos donde se encontró que a mayor glucemia en el momento de la amputación, mayor es la probabilidad de muerte a 5 años $(p=0.015)$. Por ello, se procedió a realizar una curva ROC (Receiver Operating Characteristic) con el objetivo de buscar a partir de qué valor de la glucemia en el momento de la amputación los pacientes tienen mayor riesgo de muerte a los 5 años, y el resultado más sensible fue $134 \mathrm{mg} / \mathrm{dl}$ (sensibilidad del 96\% y
Tabla 2. Coordenadas de la curva ROC (Receiver Operating Characteristic) y valor de la última glucemia antes de la amputación

\begin{tabular}{|c|c|c|}
\hline Última glucemia (mg/dl) & Sensibilidad & 1 - especificidad \\
\hline 107 & 1 & 1 \\
\hline 134 & 0.963 & 0.9 \\
\hline 165 & 0.926 & 0.7 \\
\hline 170.5 & 0.926 & 0.6 \\
\hline 175.5 & 0.889 & 0.6 \\
\hline 190 & 0.704 & 0.4 \\
\hline 205 & 0.519 & 0.3 \\
\hline 211 & 0.444 & 0.3 \\
\hline 213.5 & 0.407 & 0.3 \\
\hline 217.5 & 0.37 & 0.3 \\
\hline 230 & 0.37 & 0.2 \\
\hline 245 & 0.37 & 0.1 \\
\hline 265 & 0.296 & 0 \\
\hline 280.5 & 0.259 & 0 \\
\hline 299 & 0.222 & 0 \\
\hline 319.5 & 0.185 & 0 \\
\hline 326.5 & 0.148 & 0 \\
\hline 390.5 & 0.111 & 0 \\
\hline 452.5 & 0.074 & 0 \\
\hline 477.5 & 0.037 & 0 \\
\hline 501 & 0 & 0 \\
\hline
\end{tabular}

especificidad del 10\%); la mejor relación entre sensibilidad y especificidad se observó para $170 \mathrm{mg} / \mathrm{dl}$ (sensibilidad del $92 \%$ y especificidad del $40 \%$ ) y 190 $\mathrm{mg} / \mathrm{dl}$ (sensibilidad del $70 \%$ y especificidad del $60 \%$ ), y el valor de mayor especificidad se observó para 265 $\mathrm{mg} / \mathrm{dl}$ (sensibilidad del 29\% y especificidad del 100\%) (Tabla 2) (Fig. 1).

\section{Discusión}

En nuestro estudio, la mortalidad a 1 año fue del $8.1 \%$, menor que la reportado en la literatura (13-40\%); a los 3 años fue del $43 \%$, lo que coincide con lo reportado en la literatura (35-65\%); y a los 5 años fue del $72.97 \%$, que también concuerda con lo reportado en la literatura $(39-80 \%)^{7,10,11}$. 


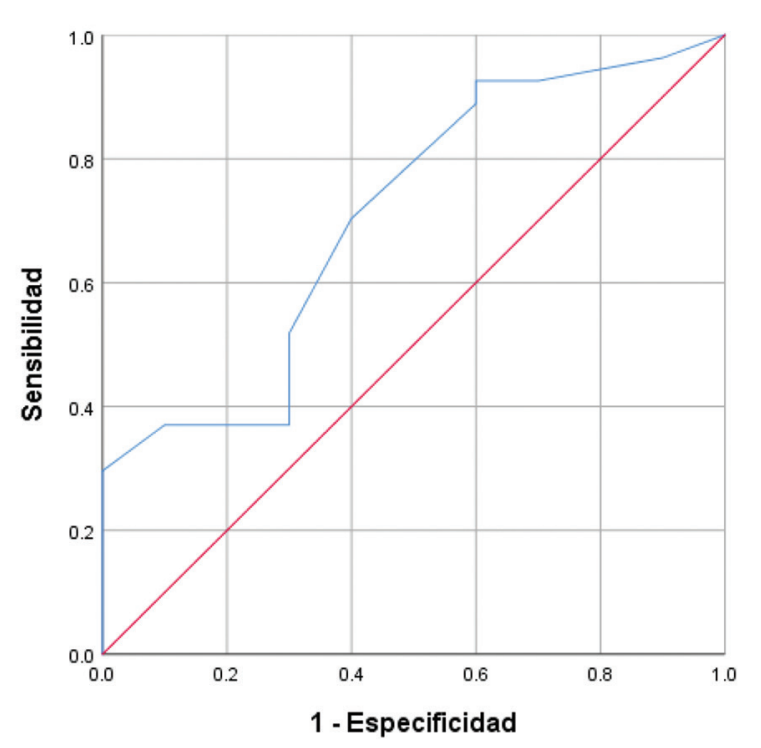

Figura 1. Curva ROC (Receiver Operating Characteristic) de mortalidad contra última glicemia tomada antes de la amputación. Los segmentos de diagonal se generan mediante empates. Área bajo la curva $=0.707$.

Se observó que el $73 \%$ de los pacientes con amputación supracondílea habían fallecido (OR: $0.72 ; p=0.275$ ); en la literatura se describe un riesgo del $61.1 \%$, con OR de 4.5 , en relación con los pacientes con amputación a otros niveles ${ }^{7}$.

El motivo de la amputación de los 37 pacientes en nuestro estudio fue la etiología infecciosa en 24, la etiología vascular en 31 y la etiología neuropática en 13 , en coincidencia con la literatura, en la que se menciona que más del $50 \%$ de los pacientes que presentan una primera amputación tienen evidencia de enfermedad arterial periférica ${ }^{6}$.

La insuficiencia cardiaca supuso un aumento del riesgo de muerte de $1.42(p=0.79)$, lo que coincide con lo reportado en la literatura, donde se señala un aumento del riesgo de muerte 1.71 veces mayor que en los pacientes sin insuficiencia cardiaca ${ }^{15}$.

De los pacientes con enfermedad renal crónica de nuestro estudio, fallecieron el $80 \%$ (OR: $1.47 ; p=0.46$ ), lo cual no coincide con la literatura, donde se refiere que fallecen el $43.6 \%$ (OR: 1.84); lo anterior debido a que no se tomó en cuenta si el paciente estaba en algún tipo de terapia sustitutiva renal ${ }^{14,15}$.

Se observó un aumento del riesgo en los pacientes con EPOC, 1.125 veces más alto que en la población sin EPOC, lo cual coincide con la literatura, que reporta 1.3 veces más riesgo.

En nuestro estudio, el infarto agudo al miocardio supone un riesgo 1.2 veces mayor que en los pacientes sin infarto, mientras que en la literatura se menciona un aumento del riesgo en los pacientes con infarto de 2.013.33 veces más que en los pacientes sin infarto.

No se han encontrado estudios que mencionen un número de enfermedades concomitantes asociadas a la amputación para aumentar el riesgo de muerte; en nuestro estudio encontramos que tres o más enfermedades se asocian a un aumento del riesgo de muerte de 1.6 veces en comparación con los pacientes que tienen dos o menos enfermedades concomitantes $(p=0.018)$.

Por último, para la glucemia determinada previamente a la amputación se demostró que, cuanto mayor era, más alta resultaba la probabilidad de muerte a 5 años $(p=0.015)$, y que la probabilidad de morir en los 5 años posteriores a la amputación al tener una glucemia de $134 \mathrm{mg} / \mathrm{dl}$ era del $10 \%$, con una glucemia de $170 \mathrm{mg} / \mathrm{dl}$ era del 40\%, con una glucemia de $190 \mathrm{mg} / \mathrm{dl}$ era del $60 \%$ y con una glucemia de $265 \mathrm{mg} / \mathrm{dl}$ era del $100 \%$.

En nuestro estudio, la causa más común de muerte fue el choque séptico, en 12 pacientes, al igual que lo reportado en los países en desarrollo ${ }^{6}$.

\section{Conclusiones}

Cuanto mayor es la glucemia en el momento de la amputación, mayor es la probabilidad de muerte a 5 años, y el riesgo de muerte es 1.6 veces mayor en los pacientes que presentan tres o más enfermedades concomitantes. Dentro de la literatura mundial existen múltiples estudios realizados en los Estados Unidos de América y en Europa, pero hacen falta estudios en México con seguimiento prospectivo, de carácter multicéntrico y con muestras heterogéneas que permitan tener un panorama nacional.

\section{Agradecimientos}

Al Hospital General de Zona No. 6, por permitirnos utilizar los recursos para la elaboración de este artículo con fines educativos.

\section{Responsabilidades éticas}

Protección de personas y animales. Los autores declaran que para esta investigación no se han realizado experimentos en seres humanos ni en animales.

Confidencialidad de los datos. Los autores declaran que han seguido los protocolos de su centro de trabajo sobre la publicación de datos de pacientes. 
Derecho a la privacidad y consentimiento informado. Los autores han obtenido el consentimiento informado de los pacientes y/o sujetos referidos en el artículo. Este documento obra en poder del autor de correspondencia.

\section{Conflicto de intereses}

Los autores declaran no tener ningún conflicto de intereses.

\section{Bibliografía}

1. Van Netten JJ, Baba M, Lazzarini PA. Epidemiology of diabetic foot disease and diabetes-related lower-extremity amputation in Australia: systematic review protocol. Syst Rev. 2017;6:1-6.

2. Gabel J, Jabo B, Patel S, Kiang S, Bianchi C, Chiriano J, et al. Analysis of patients undergoing major lower extremity amputation in the Vascular Quality Initiative. Ann Vasc Surg. 2018;46:75-82.

3. Hoffstad O, Mitra N, Walsh J, Margolis DJ. Diabetes, lower-extremity amputation, and death. Diabetes Care. 2015;38:1852-7.

4. Everett $\mathrm{E}$, Mathioudakis N. Update on management of diabetic foot ulcers. Ann N Y Acad Sci. 2018;1411:153-65.

5. Faglia E, Clerici G, Clerissi J, Gabrielli L, Losa S, Mantero M, et al. Long-term prognosis of diabetic patients with critical limb ischemia: a population-based cohort study. Diabetes Care. 2009 May;32(5):822-7.
6. Morbach S, Furchert H, Gröblinghoff $U$, Hoffmeier $H$, Kersten $K$, Klauke GT, et al. Long-term prognosis of diabetic foot patients and their limbs: amputation and death over the course of a decade. Diabetes Care. 2012;35:2021-7.

7. Cardoso NA, Cisneros L de L, Machado CJ, Procópio RJ, Navarro TP. Risk factors for mortality among patients undergoing major amputations due to infected diabetic feet. J Vasc Bras. 2018;17:296-302.

8. Ascencio-Montiel I de J. 10 years analysis of diabetes-related major lower extremity amputations in Mexico. Arch Med Res. 2018;49:58-64.

9. Evans KK, Attinger CE, Al-Attar A, Salgado C, Chu CK, Mardini S, et al. The importance of limb preservation in the diabetic population. J Diabetes Complications. 2011;25:227-31.

10. Beyaz S, Güler ÜÖ, Bağır GŞ. Factors affecting lifespan following below-knee amputation in diabetic patients. Acta Orthop Traumatol Turc. 2017;51:393-7.

11. Kennedy GEM, McGarry K, Bradley G, Harkin DW. All-cause mortality amongst patients undergoing above and below knee amputation in a regional vascular centre within 2014-2015. Ulster Med J. 2019;88: 30-5

12. Al-Rubeaan K, Al Derwish M, Ouizi S, Youssef AM, Subhani SN, Ibrahim HM, et al. Diabetic foot complications and their risk factors from a large retrospective cohort study. PLoS One. 2015;10:1-17.

13. Franz D, Zheng Y, Leeper NJ, Chandra V, Montez-Rath M, Chang TI. Trends in rates of lower extremity amputation among patients with end-stage renal disease who receive dialysis. JAMA Intern Med. 2018;178:1025-32

14. Hickson LJ, Rule AD, Thorsteinsdottir B, Shields RC, Porter IE, Fleming MD, et al. Predictors of early mortality and readmissions among dialysis patients undergoing lower extremity amputation. J Vasc Surg. 2018;68:1505-16

15. Stern JR, Wong CK, Yerovinkina M, Spindler SJ, See AS, Panjaki S, et al. A meta-analysis of long-term mortality and associated risk factors following lower extremity amputation. Ann Vasc Surg. 2017;42: 322-7. 